\title{
Surgical treatment and long-term outcomes of low-grade myofibroblastic sarcoma: a single- center case series of 15 patients
}

\author{
Jong-Ho Kim', Woosuk Choi ${ }^{1}$, Hwan Seong Cho², Kyu Sang Lee ${ }^{3}$, Joseph Kyu-hyung Park ${ }^{1}$ and Baek-Kyu Kim ${ }^{\text {** }}$
}

\begin{abstract}
Background: Low-grade myofibroblastic sarcoma (LGMS) is a poorly studied, rare, soft tissue sarcoma. LGMS is characterized by a low malignancy potential, tendency for local recurrence, and low likelihood of distant metastases. However, no studies have reported on the surgical treatment method and its long-term outcomes.

Methods: We included all patients treated for LGMS at our institution between March 2010 and March 2021. Medical charts were retrospectively reviewed to collect demographic information, as well as information about the clinical course, tumor characteristics, and outcomes. Statistical analysis was performed to identify the factors associated with the recurrence rate.

Results: Fifteen patients who underwent surgical treatment were enrolled in this study. There were seven cases in the upper extremities, four in the trunk area, three in the lower extremities, and one in the head and neck area. There were no metastatic cases and two cases of local recurrence.

Conclusions: The incidence of LGMS in the extremities or trunk may be higher than expected based on the current literature. Univariate analysis showed that local tissue invasion and surgical method could be associated with local recurrence. Although further large studies are needed to establish risk factors of local recurrence or extent of resection margins, based on our study, wide local excision under the proper diagnosis is the most important treatment.
\end{abstract}

\section{Introduction}

Low-grade myofibroblastic sarcoma (LGMS) is an extremely rare type of malignant tumor that was first reported by Mentzel et al. [1] These tumors originate from the mesenchyme and show myofibroblastic proliferation with fibromatosis-like features [2]. LGMS was first classified as a new group of soft tissue and bone tumors by the World Health Organization in 2002, and this classification was maintained in 2013 [3]. LGMS is

\footnotetext{
*Correspondence: plasrecon@gmail.com

${ }^{1}$ Department of Plastic and Reconstructive Surgery, Seoul National University College of Medicine, Seoul National University Bundang Hospital, 82 Gumi-ro 173beon-gil, Bundang-gu, Seongnam 463-707, Korea

Full list of author information is available at the end of the article
}

characterized by a low malignancy potential, tendency for local recurrence, and low likelihood of distant metastases $[1,4]$. LGMS has been reported to occur in deep soft tissues of the head and neck region, extremities, trunk, and abdominal cavities [5, 6]. However, LGMS has also been reported in other locations such as the posterior chest wall [7], breasts [2], vulva [8], and fingers [9]. Only a few clinical studies of this disease have been conducted so far, and there is still no consensus on the optimal surgical method or standard treatment [10]. To the best of our knowledge, no previous case series has reported surgical methods and their long-term outcomes. Here, we report surgical methods used to treat patients with LGMS and their long-term outcomes to inform clinical treatment of LGMS. original author(s) and the source, provide a link to the Creative Commons licence, and indicate if changes were made. The images or other third party material in this article are included in the article's Creative Commons licence, unless indicated otherwise in a credit line to the material. If material is not included in the article's Creative Commons licence and your intended use is not permitted by statutory regulation or exceeds the permitted use, you will need to obtain permission directly from the copyright holder. To view a copy of this licence, visit http://creativecommons.org/licenses/by/4.0/. The Creative Commons Public Domain Dedication waiver (http://creativeco mmons.org/publicdomain/zero/1.0/) applies to the data made available in this article, unless otherwise stated in a credit line to the data. 


\section{Methods}

We investigated all patients surgically treated for LGMS at our institution between March 2010 and March 2021. This study was approved by the Ethics Committee of Seoul National University Bundang Hospital. All clinical information, including sex, age, location, size, and followup period, was retrospectively collected. Surgical methods, histopathological findings, and treatment outcomes were retrospectively reviewed (Table 1).

\section{Surgical method}

The primary tumor was widely excised to achieve a negative surgical margin. In all cases, negative margins were confirmed using intraoperative frozen biopsy. The routine resection margin was $3 \mathrm{~cm}$ but adjusted according to the location of the tumor or the surrounding structures. The defect site was reconstructed by primary closure, local flap, skin graft, or free flap, based on the defect size and tumor location.

\section{Histopathological analysis}

Diagnosis was based on the histopathological features. Each case was analyzed using routine hematoxylin and eosin staining. For further characterization of the lesions, immunohistochemical studies were performed for all cases using the following stains: smooth muscle actin, desmin, beta-catenin, and Ki67. In cases referred from another hospital, the histopathological results performed at the other hospital were confirmed preoperatively at the Pathology Department of our institution. Tumor grade was determined using the Federation Nationale des Centres de Lutte le Cancer system.

\section{Statistical analysis}

Overall, local recurrence-free survival was calculated using the Kaplan-Meier method. Univariate analysis was performed by log-rank analysis to assess the factors associated with recurrence rate. Differences were considered significant at $p<0.05$. A multivariate analysis using the Cox proportional hazards regression model was not performed due to the small sample size. SPSS (version 22.0; SPSS Inc., Chicago, IL, USA) was used to perform all statistical analyses.

\section{Results}

Fifteen patients who were surgically treated and documented at our center were included. The study group comprised eight men and seven women with an average age at diagnosis of 50.8 years (range, $20-71$ years). The follow-up period ranged from 10 to 61 months (mean, 27.1 months). The primary tumors were located in the upper extremity $(n=7)$, trunk $(n=4)$, lower extremity $(n=3)$, and head and neck $(n=1)$. Seven patients were primary cases and eight patients were referred cases who underwent primary simple excision in another hospital. The average largest diameter was $3.1 \mathrm{~cm}$ (range, 0.7-5.9 $\mathrm{cm}$ ). Wide excision was performed in 13 patients, and en-bloc excision including surrounding normal tissue was performed in two patients. Negative margins were confirmed intraoperatively in the resection margins of all patients. There were four cases of local tissue invasion, including three cases of muscle and one case of bone. The average resection margin was $2.54 \mathrm{~cm}$. There were no metastatic cases and two cases of local recurrence occurred at 8 and 12 months, respectively. Chemotherapy and radiotherapy were not administered to any patient. In the log-rank analysis, local recurrence was associated with the surgical method and local tissue invasion, which were significant ( $p=0.002$ and $p=0.014$, respectively). However, a multivariate analysis was not performed due to the small sample size. The two cases of recurrence are summarized below.

\section{Case 1}

A 45-year-old female patient presented to our department with a complaint of an enlarging lesion in her upper abdomen. Physical examination revealed a tender mass, approximately $4 \times 4 \mathrm{~cm}$ in size (Fig. 1). Under suspicion of lymphoma following abdominal computed tomography, a core needle biopsy was performed, and a spindle cell proliferative lesion was identified. Chest wall magnetic resonance imaging (MRI) showed a lobulated mass $(4.1 \times 3.8 \times 4.8 \mathrm{~cm})$ in the left paramedian anterior chest wall (Fig. 2). The lesion was resected, including as much of the surrounding tissues as much as possible. However, it was not possible to obtain a sufficient resection margin due to the location of the lesion. Tumor recurrence was suspected 8 months after the surgery due to the presence of a palpable mass, and therefore, an excision was performed. The recurrent lesion was resected with clear margins, and no further recurrence was observed 22 months postoperatively.

\section{Case 2}

A 27-year-old male patient was admitted to our department with a $1.5-\mathrm{cm}$ painless mass on the right third metacarpal joint that occurred 3 months previously. X-ray revealed bond defect of right third proximal phalanx (Fig. 3). MRI of the finger confirmed the presence of a mass $(1.9 \times 1.8 \times 1.9 \mathrm{~cm})$ encircling the third flexor digitorum tendon, and bony invasion was observed (Fig. 4). LGMS was confirmed by an incisional biopsy (Fig. 5). The lesion, including the surrounding tissue, was excised intraoperatively and the neighboring nerves and vessels were saved. The phalangeal bone was reconstructed using an iliac bone graft. Twelve months after surgery, a locally 


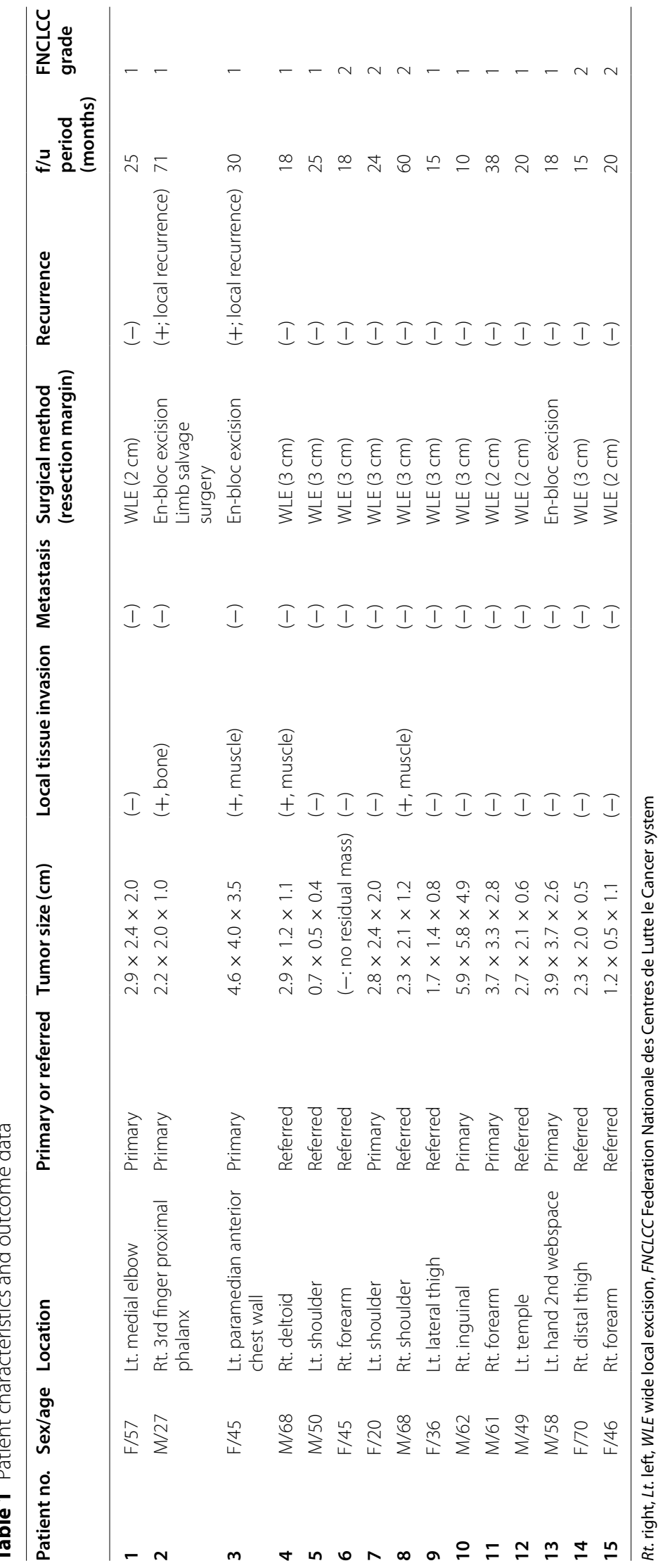




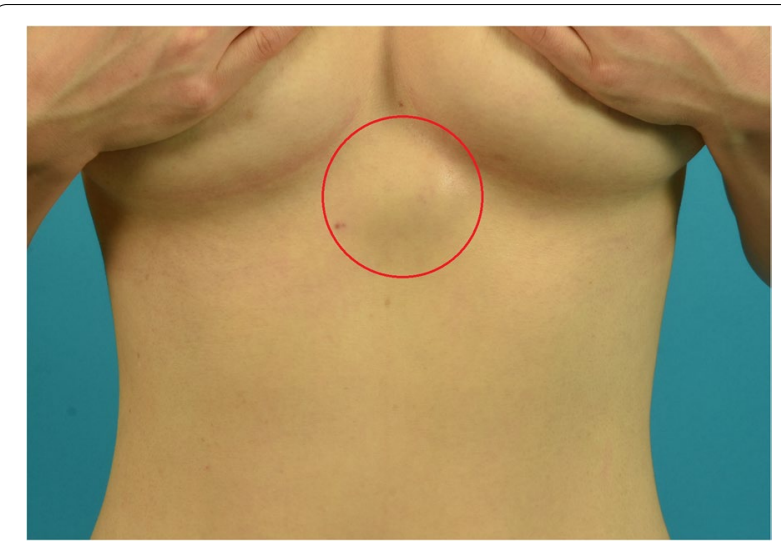

Fig. 1 Physical examination revealed a tender mass in upper abdomen, approximately $4 \times 4 \mathrm{~cm}$ in size

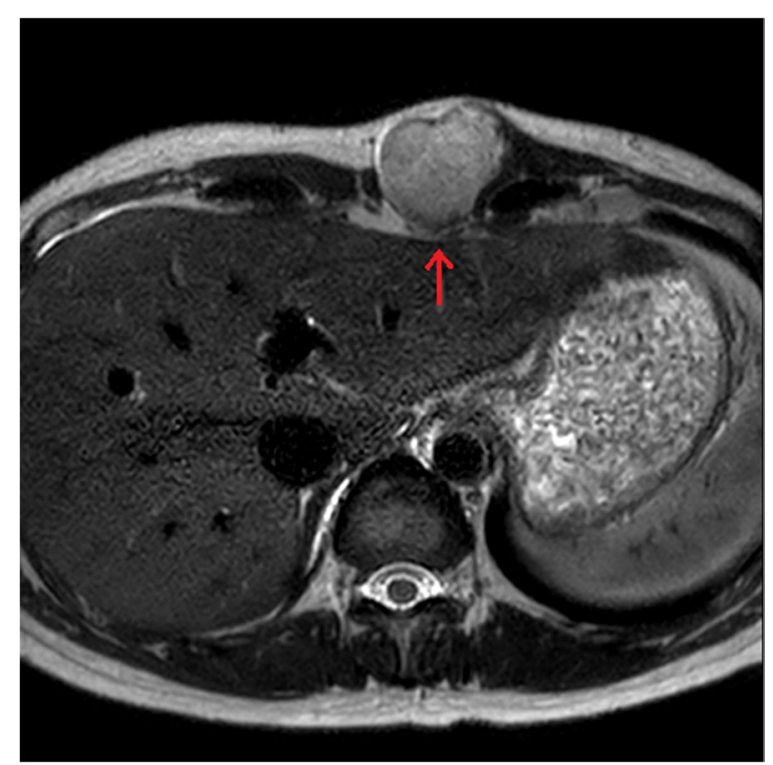

Fig. $2 \mathrm{MRI}$ showed a lobulated mass $(4.1 \times 3.8 \times 4.8 \mathrm{~cm})$ in the left paramedian anterior chest wall. MRI revealed the invasion of rectus abdominis muscle (red arrow)

palpable mass was identified, and re-excision was performed with clear margins. No further recurrence was observed 59 months postoperatively.

\section{Discussion}

LGMS is an extremely rare type of tumor that occurs primarily in adult patients with a slight male predominance. The rarity of this type of tumor makes it difficult to draw definitive conclusions regarding clinical characteristics, prognostic factors, and appropriate treatment. The

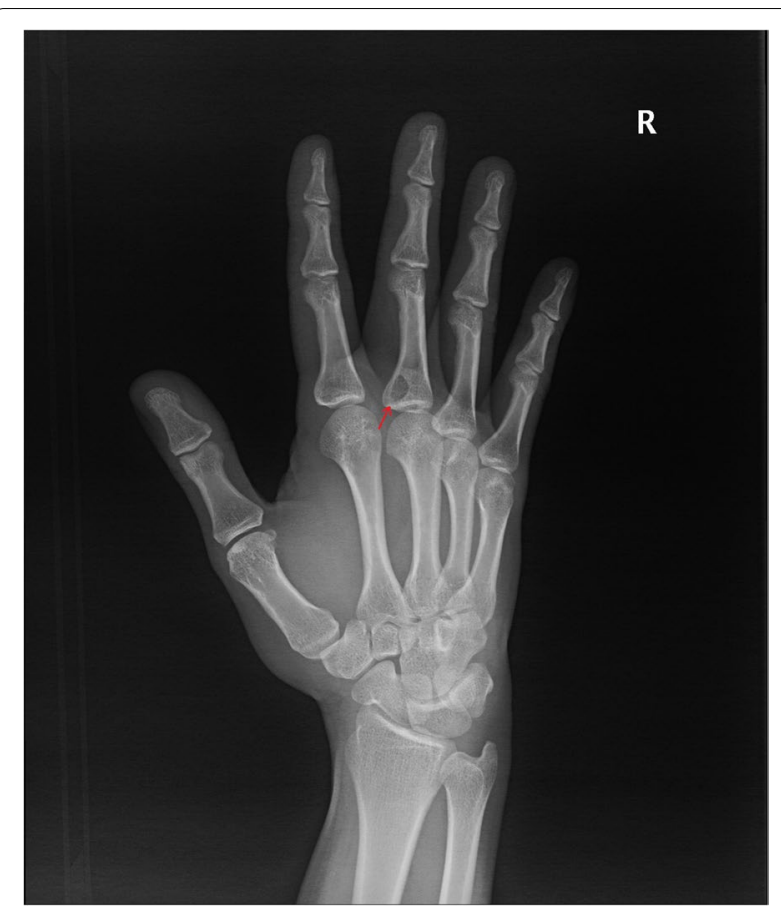

Fig. 3 X-ray revealed a bony defect of right third proximal phalanx (red arrow)

present study is one of the largest series based on data from a single institute.

\section{Clinical characteristics and prognostic factors for local recurrence}

The two largest clinical studies of LGMS were those of Mentzel et al. (18 patients) and Montgomery et al. (15 patients) [1,11]. LGMS most commonly occurs in the head and neck region with the most common site of occurrence being the oral cavity, especially the tongue [6]. Contrary to previous case reports and case series that reported the oral cavity as one of the most common sites of occurrence in the head and neck region, there were no such cases in our study. Although there is a possibility of selection bias due to the retrospective nature of our selection process, this suggests the possibility that the incidence of LGMS in the extremities or trunk is higher than previously reported. A recent population-based study in the USA reported 49 cases of LGMS with a 5-year overall survival rate of $71.6 \%$ [12]. In this population-based study, contrary to previous reports, LGMS occurred most commonly in the extremities (40.8\%), followed by the head and neck region (26.5\%). In our study, the extremities were the most common sites $(66.6 \%)$, followed by the trunk (26.7\%), and head and neck (6.7\%). In our study, a multivariate analysis was not performed due to the 


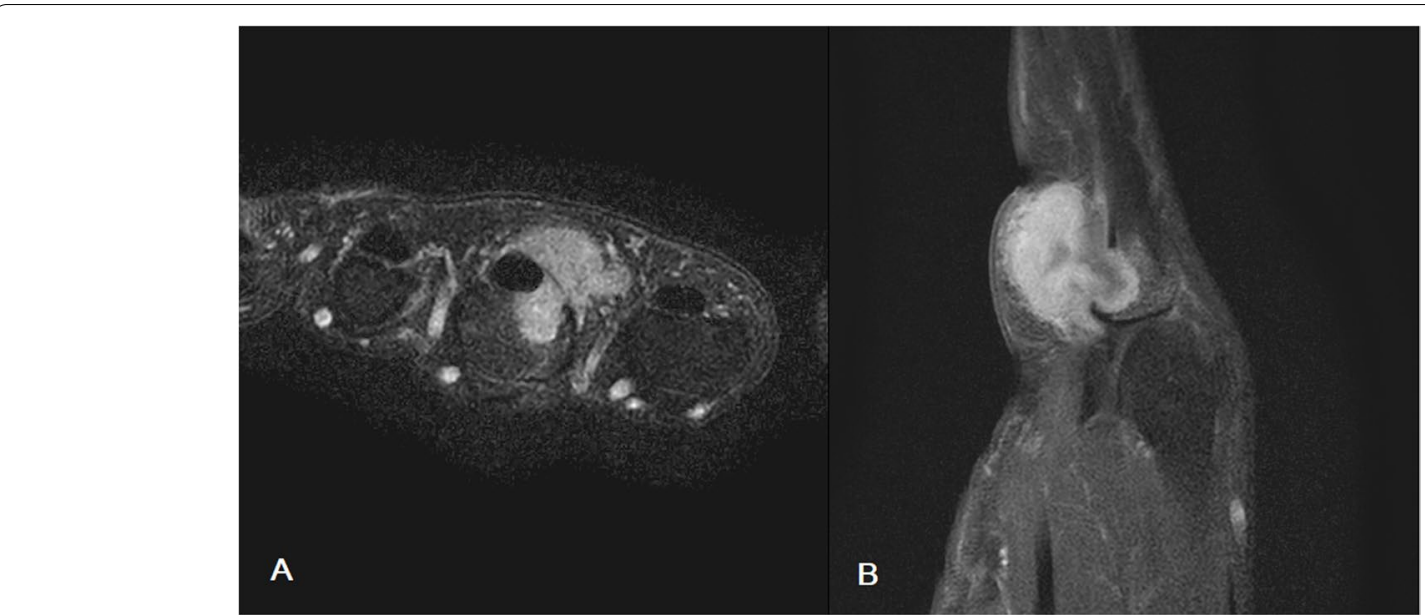

Fig. $4 \mathrm{MRI}$ of the finger confirmed the presence of a mass $(1.9 \times 1.8 \times 1.9 \mathrm{~cm})$ encircling the third flexor digitorum tendon, and bony invasion was observed. A Axial view (T2-weighted image). B Sagittal view (T1-weighted, fat suppressed image)

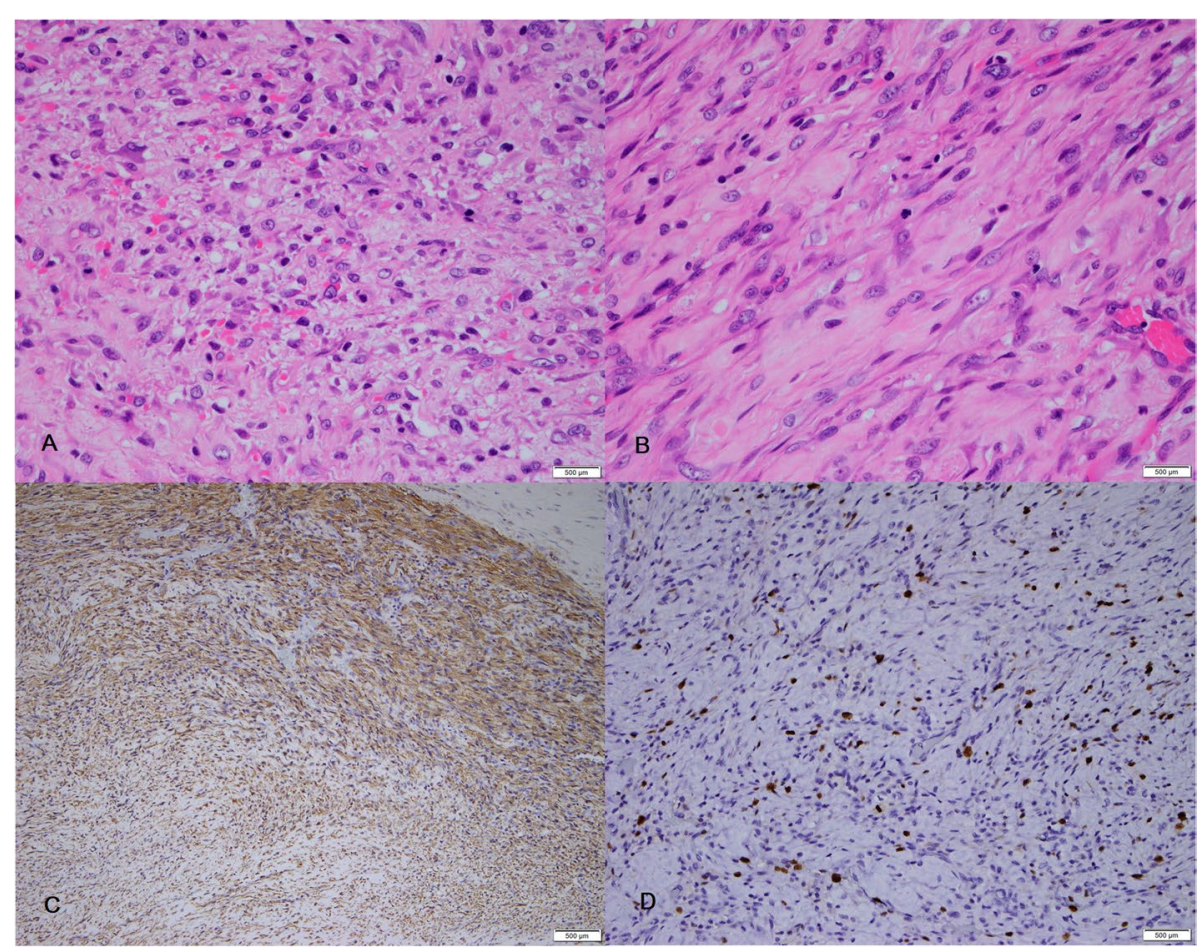

Fig. 5 Histopathologic finding. A Hematoxylin and eosin staining $(\times 40)$ showing hypercellular areas with a proliferation of spindle cells. B Hematoxylin and Eosin staining (x100) showing interlacing fascicles of spindle shaped tumor cells. C Immunohistochemistry showing positive staining for smooth muscle actin. D Positivity for Ki-67 staining was noted in more than $10 \%$ of the tumor cells

small number of patients; however, univariate analysis showed the possibility that local tissue invasion and surgical method were associated with local recurrence. The reason why the local recurrence rate was lower in our study than in previous studies is that sufficient resection margins could be achieved due to the location of the tumors in the extremities and trunk. In the previous reports, tumors were located in the head and neck, including the oral cavity, where it is difficult to obtain sufficient resection margins $[5,13]$. Some studies have reported that one-third of LGMS cases in the head and neck have a higher risk of local recurrence and 
metastasis than cases in other regions, which supports the results of our study [5].

\section{Standard treatment of LGMS}

Due to the rarity of LGMS, its optimal treatment remains unclear. In two previous studies reporting 18 and 15 patients with LGMS, only four and three patients, respectively, received chemotherapy or radiation $[1,11]$. The roles of radiotherapy and chemotherapy in LGMS remain unclear and there is no guideline to apply these treatments [12]. In some case reports, adjuvant chemotherapy showed significant clinical improvement in the presence of metastases, which was associated with prolonged progression-free survival [7]. The current study of $\mathrm{Xu} \mathrm{Y}$ et al. investigated the role of chemotherapy and radiotherapy in treating LGMS and included 96 patients from the Surveillance, Epidemiology, and End Results databas e[14]. They concluded that chemotherapy and radiation played limited roles in the outcomes of LGMS and should not be included in the standard treatment. Although successful control of LGMS was possible without chemotherapy or radiotherapy in all our cases, the treatment options depend on the location of tumor which is easily excision or not.

Resection margins are generally accepted as predictive factors for local recurrence; however, the width of resection margins remains controversial, even for other soft tissue sarcomas [15]. In our study, negative margins were confirmed in all patients, and no chemotherapy or radiation therapy was administered. In all cases with resection margins $\geq 2 \mathrm{~cm}$, no recurrence was observed during the follow-up period. There were only two cases of local recurrence without metastasis, and they were successfully controlled by local re-excision during the follow-up period. Two local recurrences occurred in patients who underwent en-bloc excision because wide local excision was difficult due to the location of the tumor (in either the paramedian chest wall or the proximal phalanx). Although resection margins were free of the tumor, it has been reported that a closer margin from the tumor may increase the risk of local recurrence [16]. Some studies have reported that a resection margin $\leq 2 \mathrm{~mm}$ was considered positive [17]. It is not yet clear whether these surgical factors influence tumor recurrence, but it is assumed that wide excision is important as a treatment for LGMS. Given these data, wide local excision, including surrounding normal tissue, seems to be the primary treatment and may become a standard treatment for management of LGMS. Although further studies are still needed to establish the optimal width or extent of resection margins, based on our study, wide local excision under the proper diagnosis is the most important treatment.

This study had several limitations. The sample size was too small for statistical analysis, and prospective studies using a larger number of patients are required. Second, as mentioned above, there is a possibility of selection bias due to the retrospective nature of the study. A large number of prospective studies are needed to properly understand the prognosis and treatment of LGMS.

\section{Conclusion}

In this study, we reported the surgical methods used to treat LGMS and the long-term outcomes of this treatment. The incidence of LGMS in the extremities or trunk may be higher in our study than has been previously reported. Factors associated with local recurrence include local tissue invasion and surgical method. We propose that wide local excision with a sufficient resection margin is important for the treatment of LGMS.

\section{Acknowledgements \\ None.}

\section{Authors' contributions}

Designing and coordinating: JK, HSC, and BK. Literature search: JK and WC. Quality assessment: JK, KSL, JKP, and BK. Data analysis and data interpretation: JK, WC, and KSL. Writing: JK, WC, and JKP. All authors have read and approved the manuscript.

\section{Funding}

None.

Availability of data and materials

Data has been anonymized and is kept with the authors. It is available upon request.

\section{Declarations}

Ethics approval and consent to participate

The study was approved by Ethics Committee (IRB no. B-2010-642-103) in the Seoul National University Bundang Hospital. As this was a retrospective chart review, the requirement to obtain individual consent was waived by the Research Ethics Board.

Consent for publication

Not applicable.

Competing interests

The authors declare that they have no competing interests.

Author details

${ }^{1}$ Department of Plastic and Reconstructive Surgery, Seoul National University College of Medicine, Seoul National University Bundang Hospital, 82 Gumi-ro 173beon-gil, Bundang-gu, Seongnam 463-707, Korea. ${ }^{2}$ Department of Orthopedic Surgery, Seoul National University College of Medicine, Seoul National University Bundang Hospital, Seoul, Korea. ${ }^{3}$ Department of Pathology, Seoul National University College of Medicine, Seoul National University Bundang Hospital, Seoul, Korea.

Received: 5 June 2021 Accepted: 23 November 2021

Published online: 07 December 2021

\section{References}

1. Mentzel T, Dry S, Katenkamp D, Fletcher CD. Low-grade myofibroblastic sarcoma: analysis of 18 cases in the spectrum of myofibroblastic tumors. Am J Surg Pathol. 1998;22:1228-38.

2. Myong NH, Min JW. Low-grade myofibroblastic sarcoma arising in fibroadenoma of the breast-A case report. Diagn Pathol. 2016;11:33. 
3. Zambo I, Vesely K. WHO classification of tumours of soft tissue and bone 2013: the main changes compared to the 3rd edition. Cesk Patol. 2014:50:64-70.

4. Qiu JY, Liu P, Shi C, Han B. Low-grade myofibroblastic sarcomas of the maxilla. Oncol Lett. 2015;9:619-25.

5. Cai C, Dehner LP, El-Mofty SK. In myofibroblastic sarcomas of the head and neck, mitotic activity and necrosis define grade: a case study and literature review. Virchows Arch. 2013;463:827-36.

6. Keller C, Gibbs CN, Kelly SM, Haller JR, White KS, Coffin CM, et al. Lowgrade myofibrosarcoma of the head and neck: importance of surgical therapy. J Pediatr Hematol Oncol. 2004;26:119-20.

7. Katalinic D, Santek F. Giant low-grade primary myofibroblastic sarcoma of the posterior chest wall. World J Surg Oncol. 2017;15:96.

8. Roth TM, Fratkin J, Woodring TC, McGehee RP. Low-grade myofibroblastic sarcoma of the vulva. Gynecol Oncol. 2004;92:361-4.

9. San Miguel P, Fernández G, Ortiz-Rey JA, Larrauri P. Low-grade myofibroblastic sarcoma of the distal phalanx. J Hand Surg. 2004;29:1 160-3.

10. Wang L, Li LX, Chen DQ, Yang L, Li SK, Cheng C. Low-grade myofibroblastic sarcoma: clinical and imaging findings. BMC Med Imaging. 2019;19:36.

11. Montgomery E, Goldblum JR, Fisher C. Myofibrosarcoma: a clinicopathologic study. Am J Surg Pathol. 2001;25:219-28.

12. Chan JY, Gooi Z, Wong EW, Ng SK, Tong MC, Vlantis AC. Low-grade myofibroblastic sarcoma: A population-based study. Laryngoscope. 2017:127:116-21.

13. Demarosi F, Bay A, Moneghini L, Carrassi A. Low-grade myofibroblastic sarcoma of the oral cavity. Oral Surg Oral Med Oral Pathol Oral Radiol Endod. 2009:108:248-54.

14. Xu Y, Xu G, Wang X, Mao M, Wu H, Baklaushev V, et al. Is there a role for chemotherapy and radiation in the treatment of patients with low-grade myofibroblastic sarcoma? Clin Transl Oncol. 2021;23(2):344-52.

15. Engellau J, Samuelsson V, Anderson H, Bjerkehagen B, Rissler P, SundbyHall K, et al. Identification of low-risk tumours in histological high-grade soft tissue sarcomas. Eur J Cancer. 2007:43:1927-34.

16. Kainhofer V, Smolle MA, Szkandera J, Liegl-Atzwanger B, Maurer-Ertl W, Gerger A, et al. The width of resection margins influences local recurrence in soft tissue sarcoma patients. Eur J Surg Oncol. 2016;42:899-906.

17. Novais EN, Demiralp B, Alderete J, Larson MC, Rose PS, Sim FH. Do surgical margin and local recurrence influence survival in soft tissue sarcomas? Clin Orthop Relat Res. 2010;468:3003-11.

\section{Publisher's Note}

Springer Nature remains neutral with regard to jurisdictional claims in published maps and institutional affiliations.

Ready to submit your research? Choose BMC and benefit from:

- fast, convenient online submission

- thorough peer review by experienced researchers in your field

- rapid publication on acceptance

- support for research data, including large and complex data types

- gold Open Access which fosters wider collaboration and increased citations

- maximum visibility for your research: over $100 \mathrm{M}$ website views per year

At BMC, research is always in progress.

Learn more biomedcentral.com/submissions 